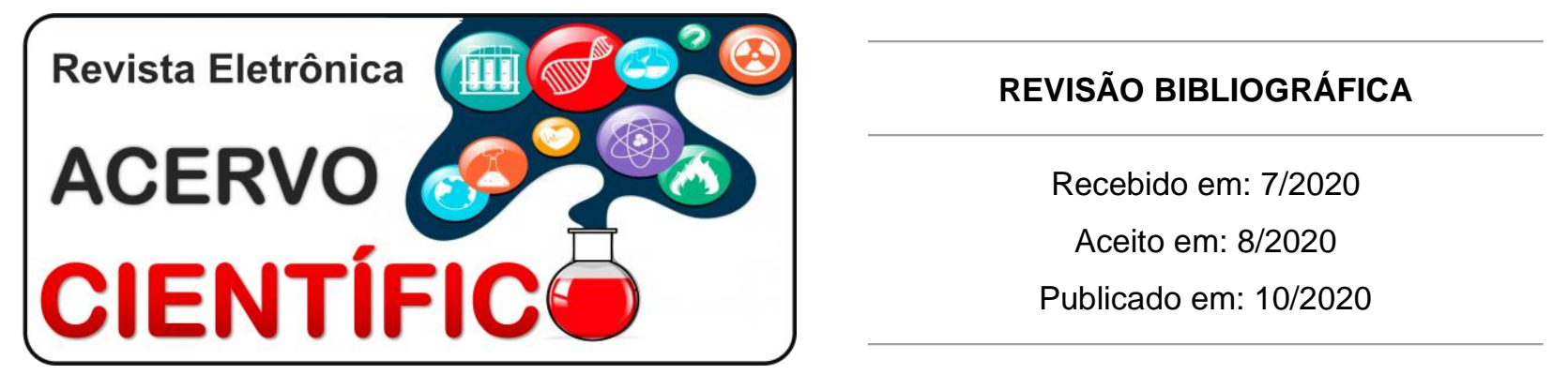

\title{
Cirurgia de revascularização do miocárdio: uma abordagem minimamente invasiva
}

\author{
Myocardial revascularization surgery: minimum invasive approach
}

\section{Cirugía de revascularización miocardial: un enfoque mínimo invasivo}

Adriana Simões Ferreira ${ }^{1 *}$, Henrique Moura Parreira1, Ariele Alves de Oliveira1, Daniela Laignier Faria $^{2}$, Izadora Helena Fonseca e Silva ${ }^{1}$, José Guilherme Reis de Oliveira ${ }^{4}$, Layra Ton ${ }^{1}$, Maria Fernanda Campos Alves ${ }^{1}$, Matheus Ribeiro Moreira ${ }^{3}$, Shesllen Mikaelly Cruz Corrêa ${ }^{5}$.

Resumo: Esse artigo buscou salientar sobre os benefícios envolvidos nas cirurgias minimamente invasivas, abordando procedimentos que visam reestabelecer o fluxo sanguíneo do miocárdio, auxiliando na sua revascularização e, assim, nas restaurações das funções efetivas do órgão. $O$ trabalho apresenta o amparo que uma cirurgia pouco invasiva traz aos pacientes, gerando uma recuperação menos agonizante e otimizando o êxito do procedimento. Além de apresentar o processo de modernização que a medicina sofreu através dos anos para alcançar modelos de procedimentos cirúrgicos que utilizam uma tecnologia de ponta em prol de um conforto maior dos pacientes que sofrem com doença arterial coronariana. Por se tratar de uma patologia que acomete, em sua maioria, um grupo de pessoas idosas, os riscos de uma cirurgia de revascularização do miocárdio pelo método convencional, aumentariam as chances de insucesso do procedimento, logo, as estratégias minimamente invasivas contribuem para que o paciente tenha complicações no pós-operatório reduzidas ou amenizadas e otimizam o prognóstico destes. Vale, ainda, ressaltar a necessidade de estudos contínuos na área que visem o aprimoramento das técnicas minimante invasivas, a ampliação do seu uso, a redução de custos e, sobretudo, o aumento de sua eficácia terapêutica.

Palavras-chave: Miocárdio, Terapêutica, Artéria torácica interna.

Abstract: This paper aims to highlight the benefits involved in minimally invasive surgeries, addressing procedures that aims to reestablish myocardial blood flow infarction, assisting in revascularization and thus restoring effective organ functions. The work presents the support that a less invasive surgery brings to the patients, generating a less agonizing recovery and optimizing the success of the procedure. In addition to presenting the modernization process that medicine has undergone over the years to achieve models of surgical procedures that use cutting-edge technology for the sake of greater comfort for patients suffering from coronary artery disease. As it is a pathology that mostly affects a group of elderly people, the risks of coronary artery bypass surgery by the conventional method, would increase the chances of failure of the

\footnotetext{
${ }^{1}$ Centro Universitário de Caratinga (UNEC), Caratinga - MG. *E-mail: adrianasimoes.f@hotmail.com

2 União Educacional do Vale do Aço (UNIVAÇO), Ipatinga - MG.

3 Universidade Federal dos Vales do Jequitinhonha e Mucuri (UFVJM), Diamantina - MG.

${ }^{4}$ Escola Bahiana de Medicina e Saúde Pública (EBMSP), Salvador - BA.

5 Faculdade Santo Agostinho (FASA), Vitória da Conquista - BA.
} 
procedure, therefore, the minimally invasive strategies, contribute to the patient may be reduced or mitigated complications after surgery and optimize the prognosis of patients undergoing this method. It is also worth emphasizing the need for continuous studies in the area, aiming at improving mini-invasive techniques, expanding their use, reducing costs and, above all, increasing their therapeutic efficacy.

Keywords: Myocardium, Therapeutics, Mammary arteries.

Resumen: Este articulo buscó señalar sobre los beneficios involucrados en las cirugías mínimamente invasivas, abordando procedimientos que tiene como objetivo restablecer el flujo sanguíneo del miocardio, ayudando en su revascularización y, así, en las restauraciones de las funciones efectivas del órgano. El trabajo presenta el amparo que una cirugía mínimamente invasiva trae a los pacientes, generando una recuperación menos agonizante y optimizando el éxito del procedimiento. Además de presentar el proceso de modernización que la medicina sofrió a través de los años para lograr modelos de procedimientos quirúrgicos que utilizan una tecnología de punta a favor de un conforto mayor de los pacientes que sufren con la enfermedad arterial coronaria. Por tratarse de una revascularización del miocardio por el método convencional, aumentarían las posibilidades de fallo del procedimiento, luego, las estrategias mínimamente invasivas, contribuyen para que el paciente tenga complicaciones en el postoperatorio reducidas 0 amenizadas y optimizan el pronóstico de los pacientes sometidos a este método. Cabe, aun, señalar la necesidad de estudios continuos en el área, con el fin de la mejora de las técnicas mínimamente invasivas, ampliación de su uso, reducción de costos y, sobre todo, aumento de su eficacia terapéutica.

Palabras clave: Miocardio, Terapéutica, Arterias mamarias.

\section{INTRODUÇÃO}

A Cirurgia de Revascularização do Miocárdio (CRM) consiste na restauração do fluxo cardíaco por meio da reconstrução das artérias coronárias com a utilização da artéria torácica interna (ou artéria mamária) ou artéria radial (MANGIONE FM, et al., 2015). Essa cirurgia é realizada quando acometimentos cardiovasculares, como a Doença Arterial Coronariana (DAC), não tem indicação de tratamento medicamentoso ou este é ineficaz. Sabe-se que a ocorrência de DAC é propiciada por sua presença no histórico familiar, dieta inapropriada, falta de exercício físico, obesidade, etilismo e tabagismo (TAKEHANA A, et al., 2019). Ademais, é notório que a presença de patologias nas artérias coronárias é menos comum em pessoas mais jovens, visto que 94 a 97\% não apresentam essa enfermidade (SARAIVA J, et al., 2016).

Além desta, existem outras indicações da CRM como a aterosclerose, isquemia extensa do miocárdio, alterações funcionais e/ou estruturais das artérias coronárias, trombose coronariana, vasoespasmo coronariano e, ainda, dor anginosa refratária não responsivo ao tratamento convencional (KNIHS NS, et al., 2017).

O procedimento cirúrgico tem o objetivo de reestabelecer o fluxo sanguíneo no local obstruído, uma vez que a baixa oferta de oxigênio e nutrientes compromete o tecido cardíaco. Além disso, a realização da CRM permite o resguardo do organismo em relação a acometimentos mais graves, como o Infarto Agudo do Miocárdio (IAM), que cursa com um quadro de necrose tecidual (FARID S, et al., 2018).

A escolha da melhor abordagem cirúrgica pode variar a partir da avaliação do paciente, como histórico patológico pregresso, fatores de riscos e diagnóstico. A CRM pode ser feita não só por meio de esternotomia mediana, que é o método mais tradicional e conservador, mas também por um método minimamente invasivo, no qual não é necessária a abertura do osso esterno e possui menor incisão (SARAIVA J, et al., 2016). Assim, tornou-se claro que os avanços tecnológicos estão cada vez mais amplos no âmbito de revascularização miocárdica, a saber, a cirurgia endoscópica e robótica são técnicas mais atuais que visam melhor desempenho e seguridade dos pacientes (SAKAGUCHI T, et al., 2020).

A revascularização do miocárdio com cirurgia minimamente invasiva (MIDCAB) tornou-se um método cirúrgico mais estabelecido após o desenvolvimento da Tomografia Computadorizada (TC) coronariana, 
pois permite um estudo anatômico coronariano com boa eficácia e reprodutibilidade (ENDO Y, et al., 2019; RODRIGUES ARV, et al., 2006).

Existem três métodos para coletar a artéria torácica interna esquerda (ATIE), a saber, os tipos de elevação, o endoscópio (bidimensional 2D ou 3D) e o sistema de cirurgia assistida por robô. Todos eles auxiliam em um pós-operatório menos doloroso, impedem uma aplicação de tensão excessiva na ferida, o que tende a facilitar a retirada do vaso para posterior enxerto (ENDO Y, et al., 2019).

Tendo em vista o que foi exposto, o presente trabalho teve como objetivo realizar uma revisão da modalidade narrativa e ratificar a relevância da abordagem cirúrgica minimamente invasiva na revascularização do miocárdio.

\section{REVISÃO BIBLIOGRÁFICA}

A CRM, também conhecida popularmente como "ponte de safena", foi desenvolvida pelo cirurgião cardiovascular doutor René Favaloro nascido em La Plata (Argentina) e, desde então, vem se consolidando como um dos procedimentos mais utilizados na revascularização do miocárdio (JONES DS, 2017). Ê um procedimento em que se pode utilizar dois tipos de enxertos, veia ou artéria do paciente para fazer a derivação da perfusão do miocárdio por meio do desvio do sangue da aorta para as artérias coronárias, técnica batizada de bypass, criando-se, assim, novos caminhos para a irrigação do miocárdio. Com isso, o coração passa a ser vascularizado pelas artérias antigas que possuem a lesão, e as novas "pontes".

Caso a artéria termine por obstruir totalmente o seu lúmen, o aporte sanguíneo se mantém pelos enxertos adicionados. Não existe um número preciso de "pontes" que podem ser feitas, o objetivo é fazer a revascularização da melhor forma para garantir o aporte sanguíneo adequado para o músculo cardíaco (NAVARRO PR, et al., 2020). Assim, essa cirurgia tem como objetivo aliviar ou resolver os sintomas de angina no peito, prevenir isquemia do miocárdio, prevenir IAM, melhorar a função ventricular, proporcionar melhor qualidade de vida física, psíquica e social do paciente, com consequente melhoria da morbidade e mortalidade dessa população (AZEVEDO DFC, et al., 2019).

Esse procedimento pode ser comparado com a Intervenção Percutânea Coronariana (IPC), também conhecida como angioplastia coronária. Essa intervenção foi utilizada pela primeira vez em 1977 e consiste em introduzir um balão na coronária obstruída, a fim de aumentar o lúmen e permitir a passagem do fluxo sanguíneo. Tal técnica, assim como a CRM, também pode alcançar a revascularização completa das artérias, porém, esse objetivo pode ser impossibilitado nessa técnica quando da existência de oclusões crônicas, doenças com anatomia coronariana complexa e riscos adicionais, como idade, arteriopatia extracardíaca e insuficiência renal (CUNHA SC, et al., 2016).

A CRM é indicada para grupos específicos de patologias, por exemplo, aterosclerose, uma doença que causa obstrução das artérias coronarianas, caracterizada pela inflamação crônica da parede dos vasos e, por consequente deposito de lipídios e tecido fibroso, causando uma possível diminuição ou até mesmo a obstrução total do fluxo sanguíneo (BERGHEANU SC, et al., 2017). Assim, essa patologia pode evoluir para a mais comum das doenças cardíacas, a DAC, que é caracterizada como uma anormalidade funcional ou estrutural das artérias coronarianas, ocasionando diminuição do aporte de oxigênio para o músculo cardíaco. Dentre os mecanismos patogênicos dessa doença, o que se destaca é a obstrução arterial, decorrente da placa aterosclerótica (BARBALHO SM, et al., 2015). Existem dois principais grupos de fatores que levam à formação dessa placa que são: os fatores modificáveis (Hipertensão Arterial Sistêmica [HAS], dislipidemia, Diabetes Mellitus [DM], tabagismo, estresse, etilismo, sedentários e obesidade), e os fatores não modificáveis (sexo, idade e fatores genéticos) (SANTANA AGC, et al., 2019).

Um dos principais tratamentos para a DAC é a CRM sem circulação extracorpórea (OPCAB), que de um modo geral, é considerada como menor incidência de complicações neurológicas, além de uma reduzida taxa de mortalidade e morbidade relacionada a grupos de alto risco e pacientes idosos (DONG $L$, et al., 2018). A OPCAB passou a ser utilizada após os meados da década de 90 , por acreditarem que diminuiria 0 número de complicações pós-operatórias. Entretanto, estudos mostram que não ocorreu diminuição 
significativa, logo, a CRM com ou sem circulação extracorpórea é igualmente segura e eficaz (SANTOS M, et al., 2018).

Além disso, comparando-se a CRM com a IPC, é perceptível que o tratamento cirúrgico pode reduzir as chances da necessidade de novos procedimentos de revascularização, possui maior eficácia na redução de sintomas anginosos e uma menor tendência de mortalidade em pacientes diabéticos. Assim, com base nesses desfechos, pode-se concluir certa superioridade da CRM em relação à angioplastia, como instrumento terapêutico da DAC (ANDRADE PJN, et al., 2018).

Outras possíveis indicações da CRM são isquemia extensa do miocárdio, decorrente da obstrução e, consequente, diminuição da irrigação do músculo cardíaco. Têm-se, também, alterações anatômicas por defeitos congênitos das artérias coronarianas, principalmente das artérias coronarianas de origem do seio caronário, o que não descarta a possibilidade de alterações no seu trajeto ou na sua estrutura. Apesar da sua incidência ser baixa e a maioria dos pacientes serem assintomáticos, é uma importante causa de arritmias cardíacas, isquemia e disfunção do miocárdio, além de morte de origem cardíaca. Outra causa é a trombose coronariana, quando o fluxo da artéria é interrompido por um coágulo. Normalmente esse coágulo tem origem da ruptura de uma placa aterosclerótica que se desloca em direção às coronárias, interrompendo, assim, o fluxo de sangue. Além disso, o vasoespasmo de uma artéria coronariana (espontaneamente ou por uso de substâncias tóxicas como a cocaína), também pode levar à redução da perfusão miocárdica. Ademais, tem-se a dor anginosa refratária após o tratamento clinico ou quando não é possível reverter o problema com métodos menos invasivos, como terapias medicamentosas e a angioplastia (KNIHS NS, et al., 2017).

A escolha da melhor abordagem cirúrgica pode variar a partir da avaliação do paciente, como histórico patológico, fatores de riscos e diagnóstico. Uma das formas da realização da cirurgia cardíaca é a esternotomia média, o procedimento mais utilizado para tal finalidade, que pode ser com ou sem circulação extracorpórea. Essa técnica, geralmente associada à circulação extracorpórea, resulta em dor significativa no pós-operatório, e uma incidência não insignificante de dor crônica e uma morbidade cirúrgica de 10 a 15\% (ALVES AFG, et al., 2012). Essa técnica foi descrita, inicialmente, em 1897, por Milton, mas só se tornou a incisão padrão nas cirurgias cardíacas em 1957, após a comprovação de superioridade sobre os outros métodos de cirurgia aberta (RESER D, et al., 2015).

A preparação desse procedimento consiste em desenhar uma linha entre a incisura jugular do esterno e seu processo xifoide, considerados pontos de referência para tal intervenção, aplicar anestesia para interromper os movimentos ventilatórios, o que reduzirá as chances de cortar a pleura durante o procedimento. Com isso, o cirurgião poderá serrar o esterno até ter acesso livre às vísceras da região, e conseguir realizar as intervenções necessárias, sempre se atentando em realizar o controle hemostático do paciente (RESER D, et al., 2015).

Sabe-se que a OPCAB foi uma otimização do método mais convencional que se dispunha, objetivando um melhor prognóstico. Contudo, em situações em que o paciente se adequa à metodologia proposta, a MIDCAB tem-se mostrado mais vantajosa em relação aos resultados de procedimentos por meio da esternotomia total. A título de exemplificação, a doença da artéria descendente anterior esquerda (DAE) propicia significativas complicações para o paciente, visto que sua dimensão e área de irrigação do miocárdio ventricular são extensas. Nesse contexto, tem-se dado preferência para o uso enxerto de bypass por meio da artéria mamária esquerda, que pode ser realizado tanto pelo OPCAB quanto pelo MIDCAB. No entanto, em casos de DAE, percebe-se que a MIDCAB é mais vantajosa, porque obtém enxerto com a mesma qualidade do método tradicional, minimiza a chance de futura revascularização do miocárdio e, no âmbito perioperatório, o paciente permanece por menos tempo na ventilação, possui menor chance de necessitar de transfusão de sangue e de cuidados na unidade de terapia intensiva. Ainda nesse cenário, nota-se que a intervenção cirúrgica minimamente invasiva é um método próspero, já que reduz significativamente a morbimortalidade no pós-operatório de cirurgias cardiovasculares e o tempo de recuperação no hospital, o que contribui não só para um tratamento mais eficaz, mas também menos oneroso (TEKIN AI e ASLAN U, 2017). 
Além disso, por ser um procedimento extremamente invasivo, a esternotomia aberta possui muitas desvantagens, dentre elas, destacando-se a dor significativa no pós-operatório. Essa dor é um problema inevitável, que pode acometer cerca de $80 \%$ dos pacientes submetidos às cirurgias e ela é percebida de forma diferenciada por cada um. Com a dissecção da ATIE, aumenta a existência significativa de dor no local. Contudo, a analgesia adequada após a cirurgia tem como consequência a diminuição de eventos adversos no pós-operatório, proporcionando um melhor conforto aos pacientes e melhorando os desfechos clínicos. Ademais, outro fator que contribui para a redução de intensidade da dor é a realização da técnica de forma adequada com consequente preservação da pleura, tendo em vista que melhora a função pulmonar e, consequentemente, exige um menor esforço de expansibilidade torácica na respiração (HUANGA APS e SAKATA RK, 2016).

A fim de solucionar ou amenizar esse problema, novas alternativas de procedimentos foram surgindo, como a cirurgia minimamente invasiva (CMI). Comparando-se os dois procedimentos, foi observado que a segunda traz muito mais benefícios que a primeira, uma vez que o paciente se recupera mais rápido no pós-operatório, sente menos dor, apresenta menor risco de infecções e sangramentos, redução da necessidade de transfusões, menor comprometimento da função respiratória e da mobilidade, menor tempo de internação na Unidade de Terapia Intensiva (UTI), alta precoce, além de trazer melhor resultado estético (SILVA JF, et al., 2020). De acordo com os estudos de Silva JF, et al. (2020), o procedimento minimante invasivo da cirurgia cardíaca pode ser feito por meio de uma minitoracotomia direita no quarto espaço intercostal, além de uma incisão periareolar para as pacientes do sexo masculino e uma incisão submamária para as do sexo feminino, esse procedimento utiliza pequenas incisões que varia de 3-6 cm ao invés de 15-30 cm como na cirurgia convencional (SILVA JF, et al., 2020).

A MIDCAB tornou-se um método cirúrgico mais estabelecido após o desenvolvimento da TC coronariana, pois permite um estudo anatômico coronariano com boa eficácia e reprodutibilidade (ENDO Y, et al., 2019; RODRIGUES ARV, et al., 2006). Além disso, pacientes pós enxertos têm menor movimentação durante o ciclo cardíaco quando comparados aos pacientes com coronárias saudáveis, sendo essa técnica de imagem uma boa opção de seguimento para pacientes revascularizados (RODRIGUES ARV, et al., 2006).

Existem três métodos para coletar a ATIE que são: tipos de elevação, o endoscópio (bidimensional 2D ou 3D), e o sistema de cirurgia assistida por robô. O método de elevação é mais convencional, é utilizado o afastador para distanciar pele, subcutâneo e músculos superficiais para colher a ATIE sob visão direta do tórax. Entretanto, ocorre uma tensão excessiva que é aplicada à ferida, levando a dor pós-operatória, atraso na cicatrização da ferida e uma dificuldade no desenvolvimento do campo de visão (ENDO Y, et al., 2019).

Um campo visual melhor pode ser encontrado através de um endoscópio 3D ou de um sistema de cirurgia assistida por robô, que proporcionam uma dor pós-operatória reduzida, facilidade na colheita da ATIE e impedem a aplicação de uma tensão excessiva na ferida. No caso da obesidade, é mais difícil a retirada da ATIE devido ao excesso de tecido adiposo. Logo, a utilização de endoscopia ou cirurgia assistida por robô, tende a facilitar essa retirada. Contudo, a diferença entre essas duas abordagens, é o custo de cada cirurgia a ser feita, tendo em vista que um sistema de cirurgia assistido por robô necessita de US \$ 1 milhão a US \$ 2.5 milhões por paciente (ENDO Y, et al., 2019).

$\mathrm{Na}$ endoscopia pode ser utilizado o endoscópio 2D ou 3D, porém, o 3D tem uma imagem esteroscópica com uma alta resolução ao ser comparada ao 2D e a possibilidade de um tempo menor para a realização do enxerto. Nessa cirurgia, para a colheita da ATIE, utiliza-se de um endoscópio e um dispositivo de eletrocautério estendido (ENDO Y, et al., 2019).

A cirurgia robótica, por sua vez, além de utilizar pequenas incisões, tem outras vantagens, por exemplo, melhora a destreza do cirurgião operador, evita tremor, permite ambidestria e melhor visualização das estruturas. Nessa modalidade, o cirurgião utilizando-se da técnica robótica consegue incisões ainda menores com cerca de $1-3 \mathrm{~cm}$, sem necessidade de abertura do esterno ou afastamento das costelas. Esse procedimento é feito por meio de um console que controla os braços do robô que são dotados de pequenos instrumentos e uma microcâmera, o que permite movimentos extremamente delicados e mais precisos do 
que as mãos de um ser humano alcançam, evitando assim, possíveis erros durante a cirurgia e melhora o campo de atuação do cirurgião (HARKY A e HUSSAIN SMA, 2019).

\section{CONSIDERAÇÕES FINAIS}

Por meio da análise bibliográfica deste estudo, fica evidente a evolução das técnicas cirúrgicas na CRM, suas indicações para o tratamento da DAC e os seus bons resultados terapêuticos frente aos tratamentos clínicos. Contudo, apesar dos excelentes resultados proporcionados pela $\mathrm{CRM}$, as abordagens cirúrgicas convencionais, como a esternotomia mediana, apresentam resultados pós-operatórios inferiores às abordagens minimamente invasiva, sobretudo no tamanho da incisão, tempo de permanência em UTI, incidência de dor pós-operatória e resultado estético final. Dessa maneira, reforça-se a necessidade de estudo contínuos na área, visando o aprimoramento das técnicas minimamente invasivas, a ampliação do seu uso, a redução de custos e, sobretudo, o aumento de sua eficácia terapêutica.

\section{REFERÊNCIAS}

1. ALVES AFG, et al. Cirurgia Robótica de Revascularização miocárdica: em busca de evidência científica no uso da tecnologia do século XXI. Rev. SOBECC, São Paulo, 2012; 17 (3): 31-42.

2. ANDRADE PJN, et al. Stents farmacológicos versus Cirurgia de Revascularização Miocárdica em Multiarteriais e Obstrução de Tronco: Meta-Análise de Ensaios Clínicos Aleatorizados. Internetional Journal of Cardiovascular Sciences, 2018; 31 (2): 152-162.

3. AZEVEDO DFC, et al. Critical analysis of the classic indications for myocardial revascularization. Rev. assoc. med. bras., 2019; 65 (3): 319-325.

4. BARBALHO SM, et al. Síndrome metabólica, aterosclerose e inflamação: tríade indissociável? Jorn. Vasc. Bras., 2015; 14 (4): 319-327.

5. BERGHEANU SC, et al. Pathophysiology and treatment of atherosclerosis. Neth Heart J., 2017; 25: $231-242$.

6. CUNHA SC, et al. Desfechos Clínicos da Intervenção Percutânea na Doença Triarterial e de Tronco da Coronária Esquerda. Internetional Journal of Cardiovascular Sciences, 2016; 29 (4): 262-269.

7. DONG L, et al. Resultados Clínicos de Curto e Médio Prazo após Revascularização Coronariana Híbrida vs. Revascularização Miocárdica sem Circulação Extracorpórea: Uma Metanálise. Arq. Bras. Cardiol., 2018.

8. ENDO Y, et al. The Utility of a 3D Endoscope and Robot-Assisted System for MIDCAB. Ann. Thorac. Cardiovasc. Surg., 2019.

9. FARID S, et al. Long-Term Outcome of Patients Undergoing Minimally Invasive Direct Coronary Artery Bypass Surgery. International Society for Minimally Invasive Cardiothoracic Surgery, 2018; 13 (1): 23-28.

10. HARKY A, HUSSAIN SMA. Robotic Cardiac Surgery: The Future Gold Standard or An Unnecessary Extravagance?. Department of Cardiothoracic Surgery, Liverpool Heart and Chest Hospital, Liverpool, United Kingdom, 2019; 34(4).

11. HUANGA APS, SAKATA RK. Dor após esternotomia - revisão. Rev Bras Anestesiol., 2016; 66 (4): 395-401.

12. JONES DS. CABG at 50 (or 107?) - The Complex Course of Therapeutic Innovation. N Engl. J. Med., $2017 ; 376$ (19): 1809-1811.

13. KNIHS NS, et al. Caminho percorrido até a cirurgia cardíaca: necessidades e expectativas no pré-operatório. Av. Enferm., 2017; 35 (1): 30-41.

14. MANGIONE FM, et al. Stents farmacológicos de segunda geração para tratamento de lesões proximais isoladas da artéria descendente anterior. Dados do Registro SAFIRA. Rev Bras Cardiol Invasiva, 2015; 23 (4): 256-260.

15. NAVARRO PR, et al. Clinical and epidemiological characteristics of acute myocardial infarction in a referral peruvian hospital. Rev. Peru Med. Exp. Salud Publica, 2020; 37 (1): 74-80.

16. RESER D, et al. Median sternotomy. Multimedia Manual of Cardio-Thoracic Surgery, 2015.

17. RODRIGUES ARV, et al. Angiografia Coronariana minimamente invasiva através de tomografia com múltiplos detectores. Arquivos Brasileiros de Cardiolgia, 2006; 86 (5).

18. SAKAGUCHI T, et al. Minimally invasive coronary artery bypass grafting: useful routine option for coronary revascularization in selected cases. The Japanese Association for Thoracic Surgery, 2020.

19. SANTANA ÁGC, et al. Fatores de risco para doença arterial coronária em docentes de uma faculdade privada do interior da Bahia. Revista de Epidemiologia e Controle de Infecção, Santa Cruz do Sul, 2019; 9 (3): 2238-3360.

20. SANTOS M, et al. Comparação dos resultados iniciais entre cirurgias de revascularização do miocárdio com e sem circulação extracorpórea. Arquivos Catarinenses de Medicina, 2018; 47 (2): 170-181.

21. SARAIVA J, et al. Coronary artery bypass surgery in young adults: excelente perioperative results and long-term survival. Centre of Cardiothoracic Surgery, 2016.

22. SILVA JF, et al. Minimally Invasive Cardiac Surgery versus Sternotomy - Pain Investigation. International Journal of Cardiovascular Sciences, 2020; 33 (1): 24-33.

23. TAKEHANA A, et al. Complicações No Pós-Operatório Imediato De Revascularização Do Miocárdio. Rev. SOBECC, 2019.

24. TEKIN AI, ARSLAN U. Perioperative outcomes in minimally invasive direct coronary artery by-pass versus off-pump coronary artery by-pass with sternotomy. Original Paper Cardiac Surgery, 2017; 12 (3): 285-290. 